\title{
A simplified numerical solution of the Miller model of secondary frost heave
}

\author{
A.C. Fowler and C.G. Noon \\ Mathematical Institute, Oxford University, 24-29 St. Giles, Oxford OXI 3LB, UK
}

(Received 15 July 1992; accepted after revision 1 February 1993)

\begin{abstract}
We describe the method used by Fowler and Krantz (1993) which reduces the Miller model of frost heave to two linear ordinary differential equations. Numerical solutions are presented and qualitative comparison is made with a numerical solution to the full system of equations given by O'Neill and Miller (1985).
\end{abstract}

\section{Introduction}

Whenever a soil is exposed to prolonged periods of sub-zero temperatures its surface may rise by as much as half a metre. This phenomenon is commonly referred to as frost heave and can be directly attributed to the accumulation of segregated seams of ice (ice lenses) that form within the soil. These ice lenses form discretely several millimetres apart and grow by imbibing water from the unfrozen soil below.

The pressures developed during the frost heave process are quite substantial and as a result the phenomenon is responsible for numerous environmental problems including damage to roads, pavements and the foundations of buildings. The formation of ice lenses gives rise to a dramatically increased (frozen) water content in the frozen soil and this leads to further problems when thawing commences. Ice lenses below the thawed region act as impermeable barriers causing the soil above to become completelely saturated and suffer a total loss of strength. Problems of this nature, costing millions of dollars each year to repair, have led to frost heave being the subject of much research since the early part of this century.
Substantial progress was made in the understanding of the frost heave process from the early empirical studies of Taber (1930) and Beskow (1935). However, because of its complexity, theoretical studies were slow in coming. Gold (1957), Penner (1959) and Everett (1961) made significant theoretical advances with the application of basic thermodynamics and the inclusion of surface tension effects. All of the aforementioned studies assume that an ice lens forms at the freezing front and are referred to as primary heave models. With this assumption it is difficult to produce a model which reflects all the characteristics of the frost heave process such as a continuous heave rate, lens initiation and extremely high heaving pressures. It is found in general that ice lenses in fact form some way behind the freezing front (see Miller, 1972) and models which include this observation are termed secondary heave models. Miller (1972, 1977, 1978, 1980) developed a model to describe secondary heave which in particular predicts much higher heaving pressures than those developed in the primary mode, predicts the formation of lenses behind the freezing front and also contains a mechanism for the initiation of a new lens.

O'Neill and Miller $(1982,1985)$ numerically 
solved the equations which describe the Miller model and found that their results reflected the heaving process quite accurately. However, because the equations needed to be solved for every lens, the algorithm required for this simulation was extremely complicated and not at all practical. Holden (1983), Holden et al. (1985) and Piper et al. (1988) simplify the model by supposing that certain fringe variables, namely temperature and pore water pressure, are quasi-static. In each of these papers a simple linear or exponential profile is used for the temperature while a consistent form for water pressure is only given in the latter paper as an exponential function of distance. Fowler and Krantz (1993) show that water pressure can be taken to be quasi-static and derive an explicit expression for its form. Using this and applying other asymptotic procedures they reduce the Miller model to two coupled ordinary differential equations for heave and frozen front depth which in fact, by a simple transformation, can be made linear. Although the work of Fowler and Krantz treats lens formation as a continuous process it is still possible to determine from their study such things as time of lens initiation, lens thickness and lens spacing.

In this paper numerical solutions of the equations found by Fowler and Krantz are presented and a qualitative comparison is made with the results of O'Neill and Miller (1985).

\section{The Miller model}

For modelling purposes a heaving soil is usually divided into three distinct regions: (1) the completely frozen soil, (2) the partially frozen soil below the lowest ice lens (frozen fringe) and (3) the unfrozen soil (Fig. 1). Because the criterion for lens formation is a mechanical condition rather than a thermal one (see below), Fowler and Krantz introduce a fourth region which lies above the lowest lens and next to the frozen fringe. This region contains ice lenses separated by partially frozen soil.

A brief description of the equations describing the Miller model is given below. (For a more complete account see for example O'Neill and Miller (1985) or Fowler and Krantz (1993).) For simplicity it is usually assumed that the soil matrix is incompres- sible. With this assumption we effectively only need consider the water and ice phases when writing down equations for the processes in the frozen fringe. Conservation of mass in this region leads to two differential equations coupled by a source term which arises because of ice production in the fringe. When considering energy conservation it is usually assumed that conduction and convection of heat are the dominant terms in the energy balance. The resulting expression is thus a parabolic equation for the temperature containing a source term which is again due to the ice production in this region. Conservation of momentum is given by Darcy's law where the hydraulic conductivity is a function of the water content. The form of this function, a large power of the water content, plays an important role in the reduction given by Fowler and Krantz. The large exponent gives rise to a boundary layer below the lowest lens and asymptotic analysis results in an explicit expression for the pore water pressure.

The temperature in the fringe is related to the water and ice pressures via a generalised form of the Clapeyron equation (Loch, 1978). The capillary equation expresses the difference in ice and water pressure as a known function of the water content. A typical function of this type, taken from O'Neill and Miller (1985), is given in Fig. 2. Similar numerical results have been produced for other forms of capillary relation taken from Black (1990).

The criterion given by Miller for the initiation of a new lens is that the stress between soil grains (effective stress) should become zero at some point within the fringe. The new lens will then form at this point. Bishop and Blight (1963) suggest that the effective stress in soil containing two permeating media is simply the difference between the overburden and a weighted average of the stresses in the permeating media. The weighting factor is called the stress partition factor and a typical example of its form, again taken from O'Neill and Miller (1985), is given in Fig. 3.

The model proposed by Miller is often called the rigid ice model of frost heave. This is due to the fact that it is assumed that the ice within the fringe moves, by a process of regelation, with a constant velocity equal to the heave rate. Fowler and Krantz argue that this hypothesis breaks down in more than one dimension and suggest an alternative form for 


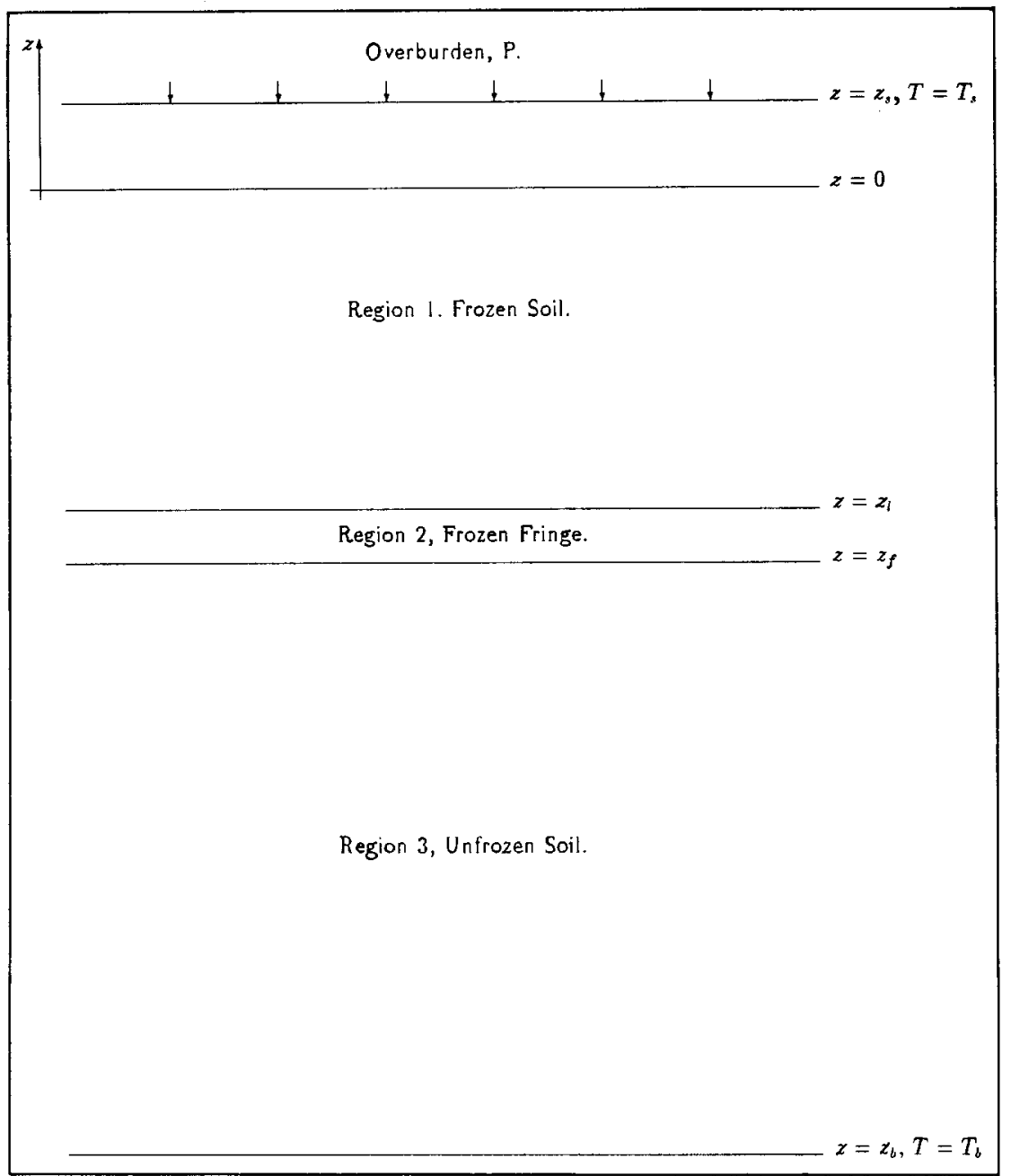

Fig. 1. Schematic picture of a heaving soil.

the ice velocity within the fringe. However, in order to give a qualitative comparison with the results of O'Neill and Miller the latter approach will not be adopted for the work presented here.

The equations applicable in regions one and three are more straight forward. We require conservation of mass, energy and momentum (Darcy's Law) in the unfrozen region while in the completely frozen zone we simply require an energy equation. Appropriate boundary conditions for each region may be found in either of the works cited above.

We now detail the observations used by Fowler and Krantz which reduce the equations describing the Miller model to two ordinary differential equa- tions. Two of these observations lead to explicit temperature profiles in regions one and three. The first is that the fringe is thin compared to the height of the soil and is consequently seen as a line by the rest of the soil. The second is that the convective transport of heat is small compared with the conductive flux (i.e. the Peclet number, $P e, \ll 1$ ). The energy equations in regions one and three then reduce to

$\nabla^{2} T=0$

in $z_{\mathrm{s}}>z>z_{\mathrm{f}}$ and $z_{\mathrm{f}}>z>z_{\mathrm{b}}$ with boundary conditions $T=T_{\mathrm{s}}$ on $z=z_{\mathrm{s}}, T=T_{0}$ on $z=z_{\mathrm{f}}$ and $T=T_{\mathrm{b}}$ on $z=z_{\mathbf{b}}$. The solution to this equation is then 
$T=T_{\mathrm{s}}+\frac{z_{\mathrm{s}}-z}{z_{\mathrm{s}}-z_{\mathrm{f}}}\left(T_{0}-T_{\mathrm{s}}\right)$

in $z_{\mathrm{s}}>z>z_{\mathrm{f}}$ and

$T=T_{0}+\frac{z_{\mathrm{f}}-z}{z_{\mathrm{f}}-z_{\mathrm{b}}}\left(T_{\mathrm{b}}-T_{0}\right)$ in $z_{\mathrm{f}}>z>z_{\mathrm{s}}$.

Using these expressions to evaluate the temperature gradient on each side of the fringe, the fringe equations may be solved to determine the locations of the moving boundaries $z_{\mathrm{s}}$ and $z_{\mathrm{f}}$. Rather than do this numerically, Fowler and Krantz use the observations given previously together with the facts that

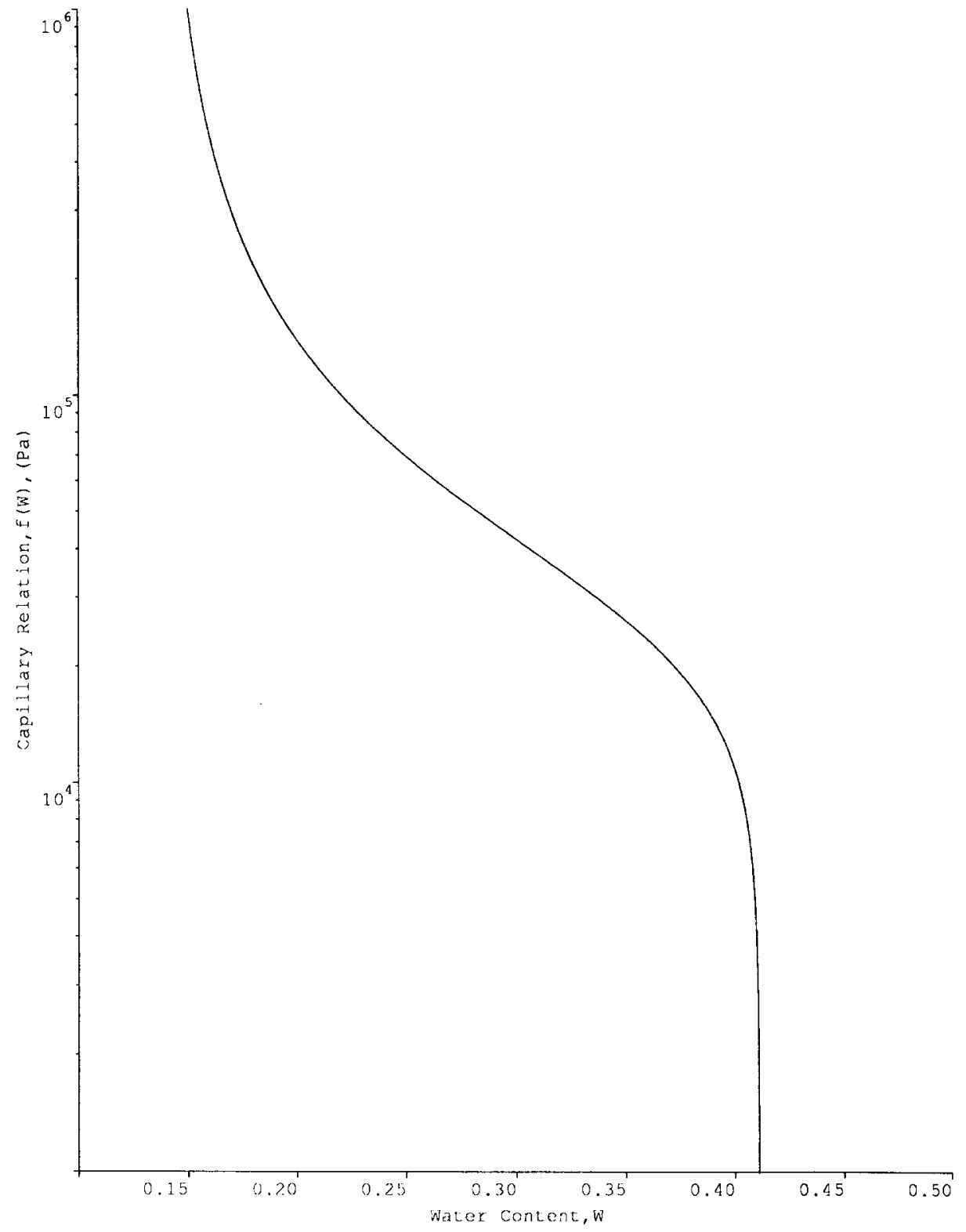

Fig. 2. Capillary relation, $f(W)$. 


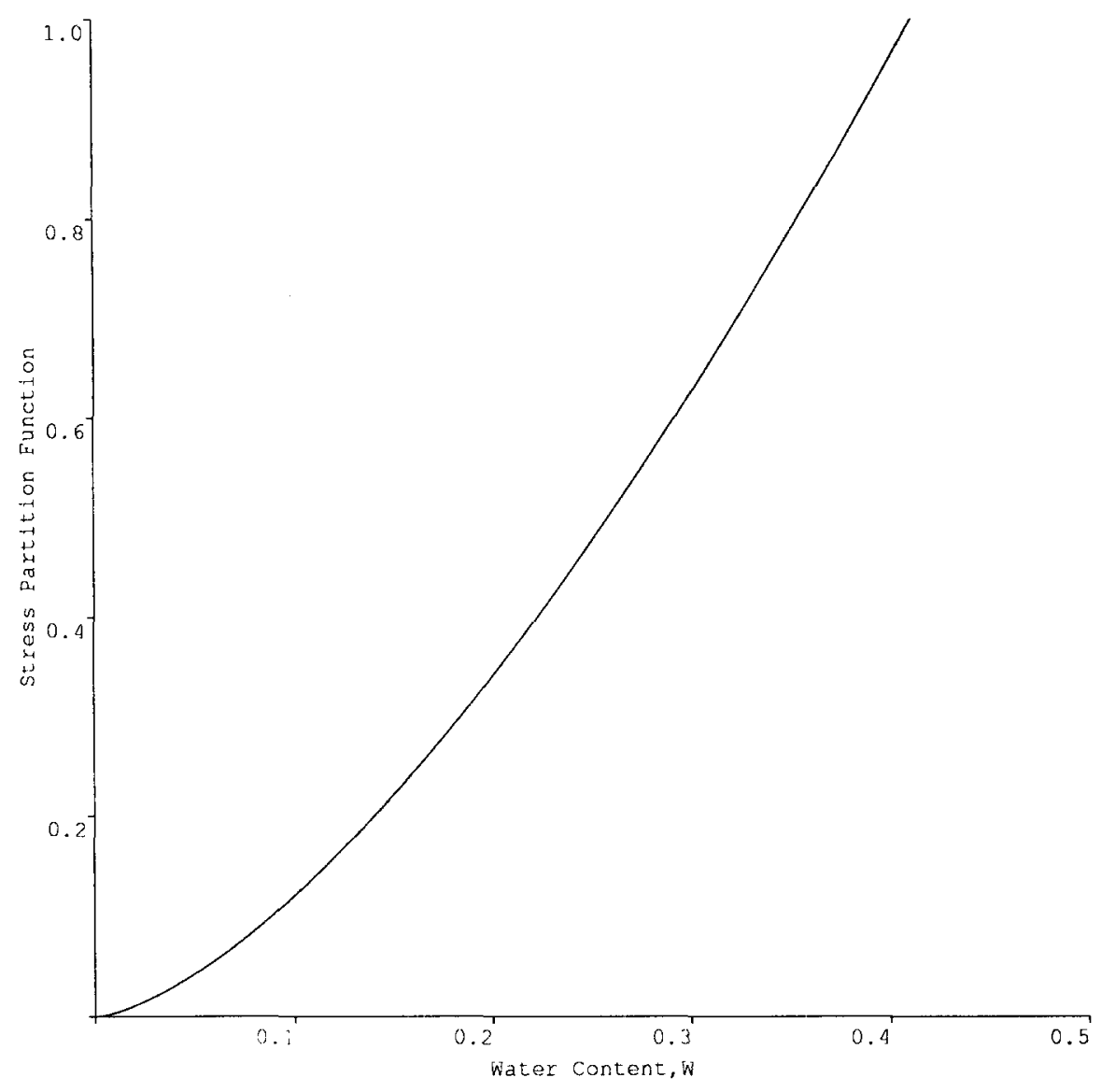

Fig. 3. Stress partition function, $\chi(W)$.

the effects of gravity are negligible and the exponent in the hydraulic conductivity function is large. This form for the hydraulic conductivity gives rise to a boundary layer below the lowest ice lens in which the water pressure changes rapidly. Asymptotic methods applied to Darcy's equation lead to a uniformly valid expression for the water pressure as a function of water content. Fowler and Krantz subsequently show that on the formation of a new lens, water content within the boundary layer relaxes rapidly to a steady state and consequently consider the water pressure to be quasi-static.

The remaining differential equations in the fringe express conservation of mass and energy. The small Peclet number and thin fringe allow these equations to be written as a set of algebraic equations for variables evaluated at each side of the fringe. Using the forms found for the temperature and pressure pro- files and also the remaining fringe relations, it is possible to reduce these algebraic equations to the two (dimensional) ordinary differential equations

$\dot{z}_{\mathrm{f}}=-\frac{A}{z_{\mathrm{s}}-z_{\mathrm{f}}}+\frac{B}{z_{\mathrm{f}}-z_{\mathrm{b}}}$
$\dot{z}_{\mathrm{s}}=\alpha\left(\frac{\nu}{z_{\mathrm{s}}-z_{\mathrm{f}}}+W_{1} \dot{z}_{\mathrm{f}}\right)$

where the constants are defined in the appendix. These constants depend upon the quasi-static water content at the base of the lowest lens. Fowler and Krantz show that lens formation can be treated as a continuous process and as a result suppose the effective pressure at the lowest lens is always zero. The existence of the boundary layer for the water pressure then enables this water content to be calculated from the stress relation. 


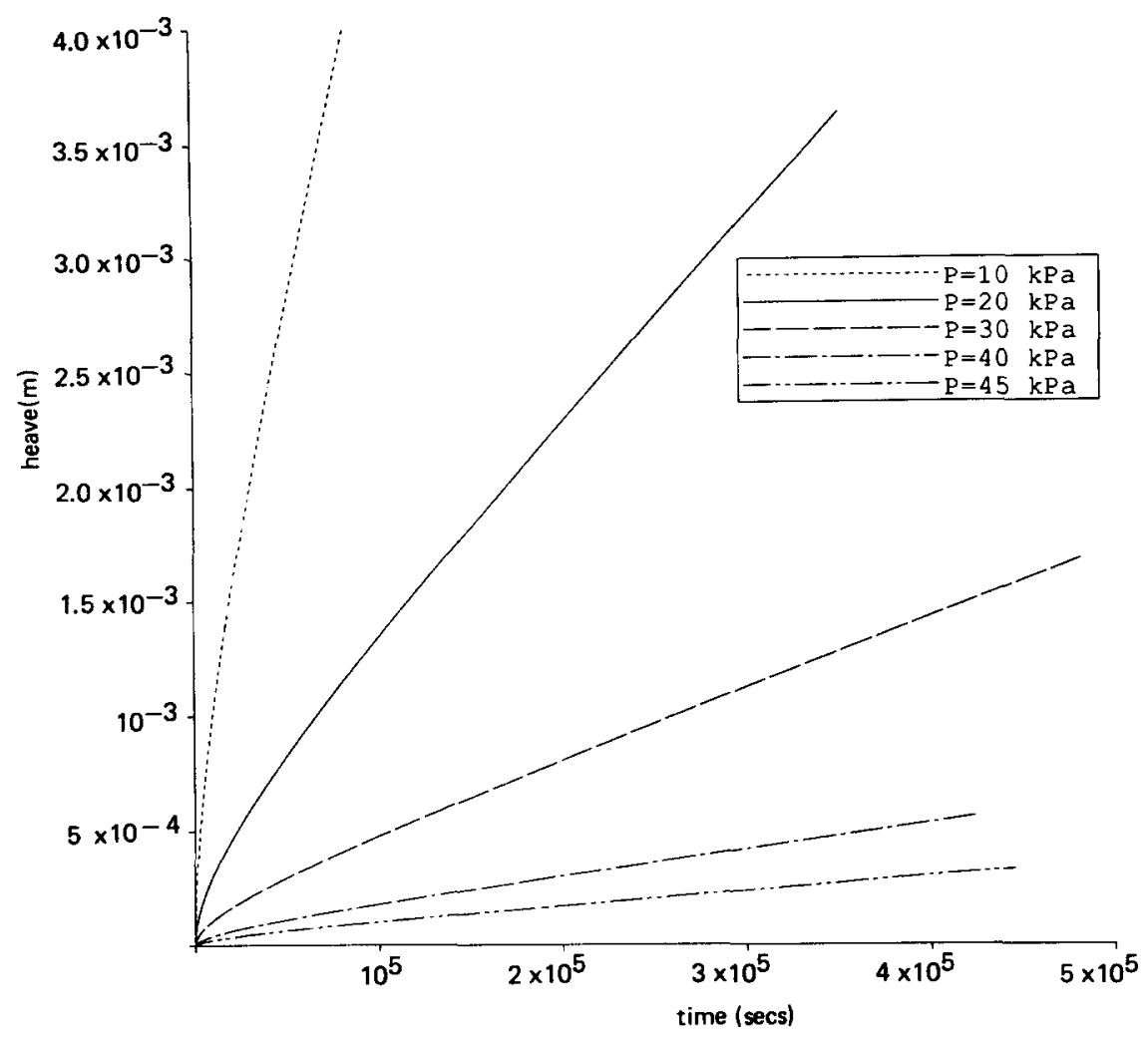

Fig. 4. Heave against time for different overburdens.

These differential equations essentially express conservation of energy across the whole fringe (Stefan condition ) and conservation of mass at the base of the lowest lens.

\section{Numerical results}

Fowler and Krantz assume that the capillary relation and its derivative have the same order of magnitude. It can be seen from Fig. 2 that this assumption may break down when the capillary suction becomes large. Most of their analysis remains valid although some care is needed when calculating the water content at the base of the lowest lens because of the rapid change in the capillary relation within the boundary layer (Noon, 1993). Calculation of this water content becomes slightly more complicated. Rather than say it is simply a zero of the stress relation we also require it to be a stationary value (see Fowler and Krantz, 1993). These conditions give two simultaneous equations for water contents at the lens and at the new lens position. Using this method, a solution is only obtained for overburdens smaller than about $50 \mathrm{kPa}$. For larger overburdens we suggest that heave is sup-

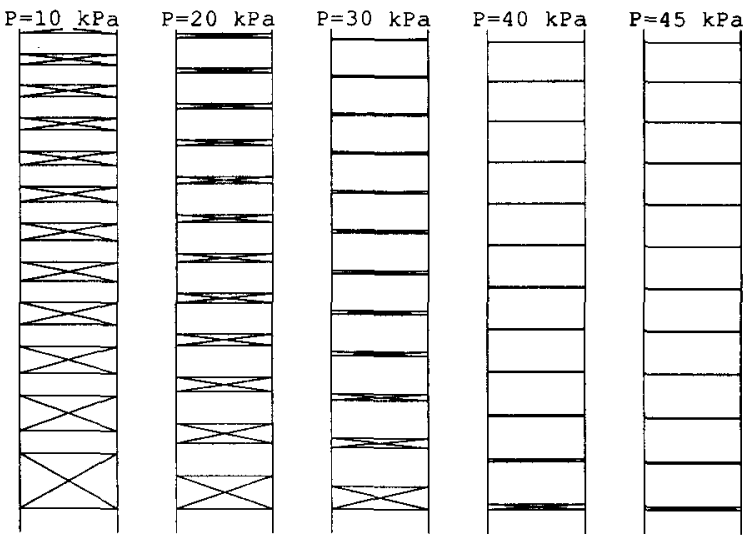

Fig. 5. Schematic sections showing twelve lenses for each overburden (crossed regions indicate lenses). 


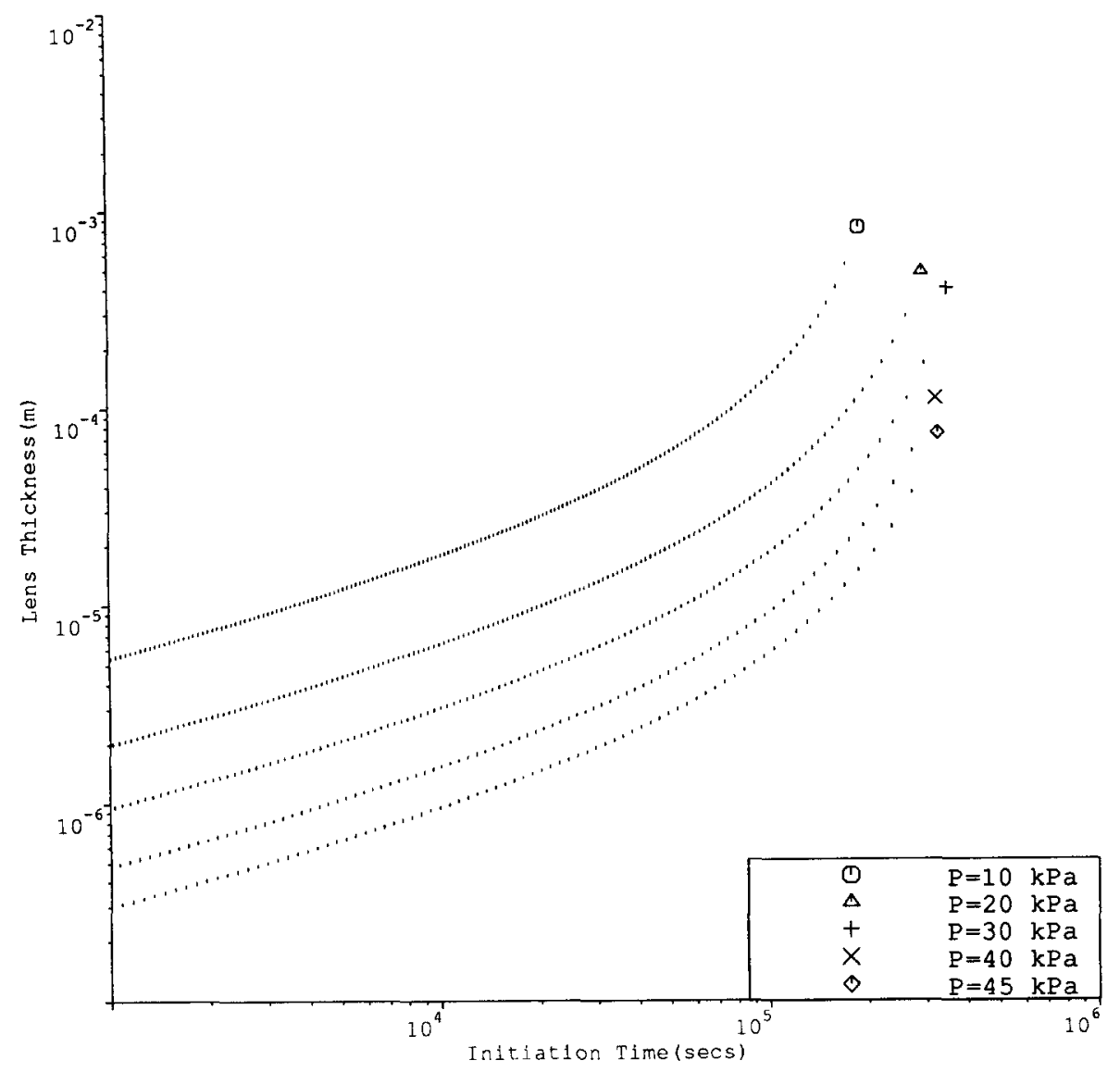

Fig. 6. Lens thickness against lens initiation time for different overburdens.

pressed for this particular capillary relation.

Equations (4) and (5) are two first order differential equations, and can be solved numerically using any standard solver, such as a Runge-Kutta method. If we write

$h_{1}=z_{\mathrm{s}}-z_{\mathrm{f}}, \quad h_{2}=z_{\mathrm{f}}-z_{\mathrm{b}}$

where $h_{1}$ and $h_{2}$ are thus the thickness of the frozen and unfrozen zones respectively, then Eqs. (4) and (5) can be written as

$\dot{z}_{\mathrm{f}}=\dot{h}_{2}=\frac{-A h_{2}+B h_{1}}{h_{1} h_{2}}$

$\dot{z}_{\mathrm{s}}-\dot{z}_{\mathrm{f}}=\dot{h}_{1}=\frac{\alpha\left(\nu h_{2}+W_{1} h_{1} h_{2} \dot{h}_{2}\right)}{h_{1} h_{2}}-\dot{h}_{2}$

( since $z_{\mathrm{b}}$ is a constant) whence $h_{1} h_{2} \dot{h}_{2}=-A h_{2}+B h_{1}$

$h_{1} h_{2} \dot{h}_{1}=\alpha \nu h_{2}+\left(1-\alpha W_{1}\right)\left(A h_{2}-B h_{1}\right)$

Now note that by defining a nonlinear transformation of time to $\tau$ defined by

$\frac{\mathrm{d} t}{\mathrm{~d} \tau}=h_{1} h_{2}$

Eqs. ( 8 ) and (9) can be written as

$\frac{\mathrm{d} h_{1}}{\mathrm{~d} \tau}=\alpha \nu h_{2}+\left(1-\alpha W_{1}\right)\left(A h_{2}-B h_{1}\right)$

$\frac{\mathrm{d} h_{2}}{\mathrm{~d} \tau}=-A h_{2}+B h_{1}$

which are a pair of linear differential equations, which can be solved exactly. By then integrating Eq. 


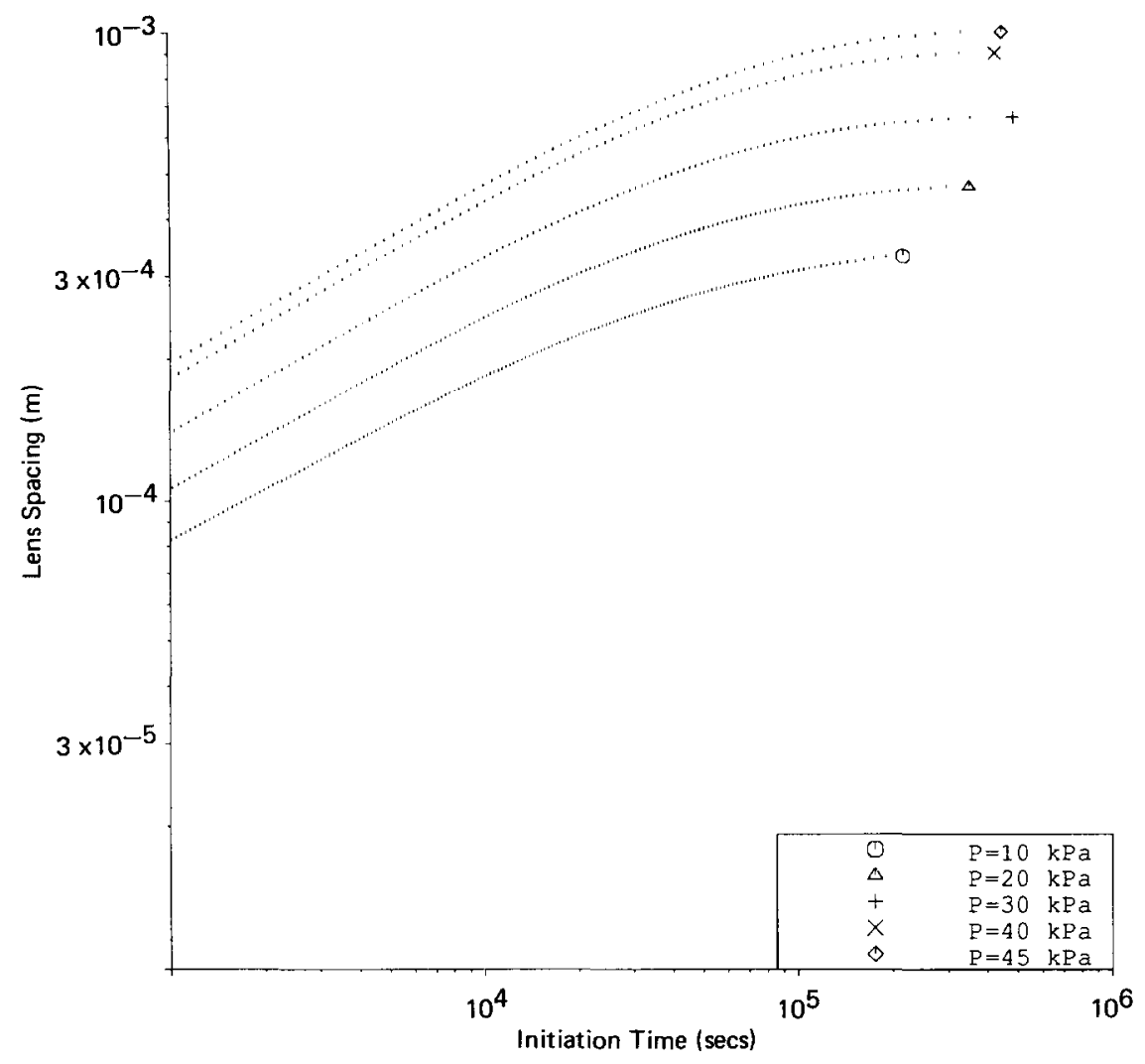

Fig. 7. Lens spacing against initiation time of following lens for different overburdens.

(10), the exact solution can be obtained in the parametric form $h_{1}=h_{1}(\tau), h_{2}=h_{2}(\tau), t=t(\tau)$.

If we consider a simple step-freeze experiment where an initially unfrozen soil is subjected to a surface temperature which is below the freezing point, then appropriate initial conditions are that $z_{\mathrm{s}}=0$, $z_{\mathrm{f}}=0$ at $t=0$. With the explicit exact solutions available, it is a straightforward matter to compute $z_{\mathrm{s}}$ and $z_{\mathrm{f}}$ as functions of time. Typical plots of heave are given in Fig. 4 for varying overburdens. These plots are only given to the time of formation of the final lens. This is because methods used to obtain the reduced model break down when lens formation ceases.

Fowler and Krantz (1993) also suggest a method for calculating lens spacing. It is then possible, from knowledge of the frozen front depth, to define an iterative procedure from which times for lens initi- ation may be found. Lens thickness may then be determined from the knowledge of the surface heave. From these calculations schematic plots of the twelve lenses above the final lens are given in Fig. 5 and plots of lens thickness and lens spacing are given in Figs. 6 and 7.

It is difficult to obtain, from the papers of O'Neill and Miller (1982, 1985), all the information required for a quantitative comparison between the full system of equations and the reduced set given here. Instead we try to give a qualitative comparison of our Figs. 4, 5, 6 and 7 with Figs. 4 and 5 from O'Neill and Miller (1985).

O'Neill and Miller's Fig. 4 shows that lens spacing tends to a constant as the final lens is approached while lens thickness increases. This is in agreement with our theory, as shown in Figs. 5, 6 and 7. Plots of heave against time are also very sim- 
ilar. For each set of plots, heave goes initially like the square root of time before becoming linear at larger times.

\section{Conclusion}

The Miller model of frost heave is the only model available which predicts all the characteristics of the frost heave process and has consequently become very popular. However, analytical solutions are not possible and because of its complexity numerical solutions are very difficult to produce. In recent years, several attempts have been made to simplify the model by "guessing" quasi-static forms for the temperature and water pressure. Fowler and Krantz (1993) derive quasi-static forms for these variables and proceed to reduce Miller's model to two ordinary differential equations. This dramatic reduction requires that: 1) the convective transport of heat may be neglected, 2 ) the effects of gravity are small, 3 ) the frozen fringe is thin and 4) the exponent in the hydraulic conductivity is large. Numerical solutions of this reduced model are very easily obtained and comparisons with numerical solutions of the full system are favourable.

We have already applied the techniques used by Fowler and Krantz to saline soils and found that the system can again be reduced to two ordinary differential equations. We hope in the future to perform similar reductions to the three dimensional problem and also to the problems of compressible and unsaturated soils.

\section{Appendix}

We define the constants required for the solution of the equations given above and give the values used in our analysis.

$z \quad=$ Displacement.

$z_{\mathrm{s}} \quad=$ Displacement of the surface.

$z_{\mathrm{f}} \quad=$ Displacement of the frozen fringe.

$z_{1} \quad=$ Position of the base of the lowest lens.

$z_{\mathrm{b}} \quad=$ Position of the base of the soil column $(-0.153 \mathrm{~m})$.

$T \quad=$ Temperature.
$T_{\mathrm{s}} \quad=$ Temperature of the soil surface $(272.5 \mathrm{~K})$.

$T_{0} \quad=$ Freezing point of water $(273 \mathrm{~K})$.

$T_{\mathrm{b}}=$ Temperature at the base of the soil column ( $274 \mathrm{~K})$.

$W=$ Water volume fraction.

$W_{1}=$ Quasi-static water content at the base of the lowest lens.

$\phi \quad=$ Pore volume fraction $(0.411)$.

$A=\frac{\nu\left(1+\delta-(1+\widetilde{\beta})\left(\phi-W_{1}\right) \alpha\right)}{\delta W_{1}+\phi+\widetilde{\beta}\left(\phi-W_{1}\right)-\alpha(1+\tilde{\beta})\left(\phi-W_{1}\right) W_{1}}$.

$B=\frac{\nu(1+\delta)(1+\beta) \theta_{\mathrm{b}}}{\delta W_{1}+\phi+\widetilde{\beta}\left(\phi-W_{1}\right)-\alpha(1+\tilde{\beta})\left(\phi-W_{1}\right) W_{1}}$.

$\alpha=\frac{\tilde{\beta}(1+\delta)}{(1+\widetilde{\beta})\left(1-\phi+W_{1}\right)}$.

$\nu=\frac{k\left(T_{0}-T_{\mathrm{s}}\right)}{L \rho_{\mathrm{w}}}$.

$\theta_{\mathrm{b}}=\frac{T_{\mathrm{b}}-T_{0}}{T_{0}-T_{\mathrm{s}}}$.

$\delta \quad=\frac{\rho_{\mathrm{w}}-\rho_{\mathrm{i}}}{\rho_{\mathrm{i}}}$.

$\tilde{\beta}=-\beta_{1}\left(\frac{f_{1}-N}{W_{1} f_{1}^{\prime}+\delta \gamma\left(f_{1}-N\right)}\right)$.

$\beta_{1}=\frac{\gamma \rho_{\mathrm{i}} L^{2} k_{0}}{g k T_{0}}\left(\frac{W_{1}}{\phi}\right)^{\gamma}$.

$f_{1} \quad=$ Capillary relation evaluated at $W_{1}$.

$f_{1}^{\prime}=$ Derivative of the capillary relation evaluated at $W_{1}$.

$N=P-p_{\infty}$.

$\rho_{\mathrm{w}}=$ Density of water $\left(1000 \mathrm{~kg} \mathrm{~m}^{-3}\right)$.

$\rho_{\mathrm{i}} \quad=$ Density of ice $\left(917 \mathrm{~kg} \mathrm{~m}^{-3}\right)$.

$g \quad=$ Acceleration due to gravity $\left(9.8 \mathrm{~m} \mathrm{~s}^{-2}\right)$.

$L=$ Latent heat of fusion for water $\left(3.35 \times 10^{5} \mathrm{~J}\right.$ $\mathrm{kg}^{-1}$ ).

$k=$ Thermal conductivity $\left(4 \mathrm{~W} \mathrm{~m}^{-1} \mathrm{~K}^{-1}\right)$.

$k_{0} \quad=$ Hydraulic conductivity of ice free soil $\left(1 \times 10^{-9} \mathrm{~m} \mathrm{~s}^{-1}\right)$.

$\gamma \quad=$ Exponent in the hydraulic conductivity function (Eq. 9).

$P \quad=$ Overburden $(10,20,30,40,45 \mathrm{kPa})$.

$p_{\infty}=$ Water pressure at $z_{\mathrm{b}}(0 \mathrm{~Pa})$.

$W_{1}$ is calculated numerically for each overburden from the equations

$$
W_{1}=\frac{\gamma\left(N-\left(1-\chi\left(W_{\mathrm{n}}\right) f\left(W_{\mathrm{n}}\right)\right)\right.}{\frac{\partial}{\partial W}[(1-\chi) f]_{W=W_{\mathrm{n}}}}
$$




$$
\begin{aligned}
& \left(1-\chi\left(W_{\mathrm{n}}\right) f\left(W_{\mathrm{n}}\right)=\right. \\
& \quad N+\left(f\left(W_{1}\right)-N\right) \exp \left(-\frac{\gamma\left(W_{\mathrm{n}}-W_{1}\right)}{W_{1}}\right)
\end{aligned}
$$

The capillary relation, stress partition and hydraulic conductivity functions used for this analysis are

$$
\begin{aligned}
& \log f(W)=0.34394 \log (0.411-W) \\
& -8.5 \times 10^{-3}(\log (0.411-W))^{2} \\
& -0.78334 \log (W-0.14) \\
& +3.91 \times 10^{-2}(\log (W-0.14))^{2} \\
& \chi(W)=\left(\frac{W}{\phi}\right)^{1.5} \\
& k(W)=k_{0}\left(\frac{W}{\phi}\right)^{9}
\end{aligned}
$$

The functional forms chosen here have been suggested by O'Neill and Miller (1985); the coefficients in $f(W)$ were determined by a least squares fit to the curve given by them.

\section{Acknowledgements}

C.G. Noon acknowledges the support of an S.E.R.C earmarked studentship on "Secondary Frost Heave in Freezing Soils". We thank Kevin O’Neill for discussion.

\section{References}

Beskow, G., 1935. Soil freezing and frost heaving with special applications to roads and railroads. Swed. Geol. Soc., Ser. C, No. 375,26 th year book, No. 3 (translated by J.O. Osterberg, Northwestern Univ., 1947). Reprinted in CCREL Spec. Rep. 91-23, 1991.
Bishop, A.W. and Blight, G.E., 1963. Some aspects of effective stress in saturated and partly saturated soils. Géotechnique, 13: 177-197.

Black, P. 1990. Three functions that model empirically measured unfrozen water content and predict relative hydraulic conductivity. CRREL Tech. Rep. 90-5.

Everett, D.H., 1961. The thermodynamics of frost damage to porous solids. Trans. Faraday Soc., 57: 1541-1551.

Fowler, A.C. and Krantz, W.B., 1993. On the O'Neill/Miller model for secondary frost heave. SIAM J. Appl. Math., to be published.

Gold, L.W., 1957. A possible force mechanism associated with freezing of water in porous materials. Highw. Res. Board Bull., 168: 65-72.

Holden, J.T., 1983. Approximate solutions for Miller's theory of secondary heave. Proc. 4th Int. Conf. Permafrost. National Academy Press, Washington, DC, pp. 498-503.

Holden, J.T., Piper, D. and Jones, R.H., 1985. Some developments of a rigid ice model of frost heave. In: S. Kinosita and M. Fukuda (Editors), Proc. 4th Int. Symp. on Ground Freezing. Balkema, Rotterdam, pp. 93-99.

Loch, J.P.G., 1978. Thermodynamic equilibrium between ice and water in media. Soil Sci., 126(2): 77-80.

Miller, R.D., 1972. Freezing and heaving of saturated and unsaturated soils. Highw. Res. Rec., 393: 1-11.

Miller, R.D., 1977. Lens initiation in secondary heaving. Proc. Int. Symp. Frost Action in Soils, Luleå, pp. 68-74.

Miller, R.D., 1978. Frost heaving in non-colloidal soils. Proc. 3rd Int. Conf. Permafrost. National Research Council of Canada, Ottawa, pp. 708-713.

Miller, R.D., 1980. Freezing phenomena in soils. In: D. Hillel (Editor), Applications of Soil Physics. Academic Press, New York, Ch. 11, pp. 254-299.

Noon, C.G., 1993. Secondary frost heave in freezing soils. Ph.D. Thesis, Oxford University.

O'Neill, K. and Miller, R.D., 1982. Numerical solutions for a rigid ice model of secondary frost heave. CCREL Tech. Rep. 82-13.

O'Neill, K. and Miller, R.D., 1985. Exploration of a rigid ice model of frost heave. Water Resour. Res., 21: 281-296.

Penner, E., 1959. The mechanism of frost heaving in soils. Highw. Res. Board Bull., 225: 1-13.

Piper, D., Holden, J.T. and Jones, R.H., 1988. A mathematical model of frost heave in granular materials. In: $\mathbf{K}$. Senneset (Editor), Proc. 5th Int. Conf. Permafrost. Tapir, Trondheim, pp. 370-376.

Taber, S., 1930. The mechanics of frost heaving. J. Geol., 38: 303-317. 